\title{
Schwannoma with features mimicking neuroblastoma: report of two cases with immunohistochemical and ultrastructural findings
}

\author{
S Bhatnagar, S S Banerjee, A R Mene, R J Prescott, B P Eyden
}

Department of

Pathology, Christie

Hospital, Manchester,

UK

S Bhatnagar

S S Banerjee

B P Eyden

Department of Pathology, Blackburn

Royal Infirmary,

Blackburn, UK

A R Mene

R J Prescott

Correspondence to:

Dr S S Banerjee, Consultant

Histopathologist, Pathology

Department, Christie

Hospital NHS Trust,

Wilmslow Road, Manchester

M20 4BX, UK.

Accepted for publication

17 June 1998

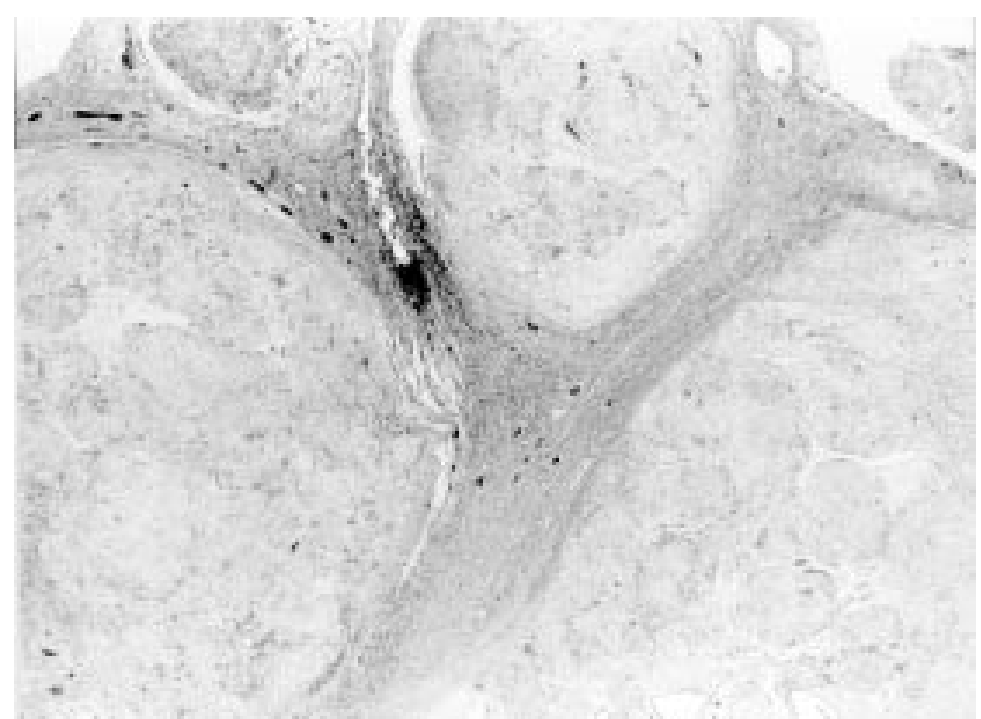

Figure 1 Low power view of a plexiform Schwannoma (case 1). Haematoxylin and eosin.

Conclusions-Benign schwannomas may contain rosette-like structures mimicking neuroblastoma/PNET. The techniques used confirmed schwannian differentiation only and eliminated neuroblastomal PNET. These uncommon variants should be recognised by practising histopatholo-

\begin{abstract}
Objective-A study of two cases of a rare variant of benign schwannoma showing areas mimicking neuroblastoma/peripheral primitive neuroectodermal tumour (PNET).

Methods-Sections of formalin fixed, paraffin embedded specimens were studied by tinctorial stains and immunohistochemistry, and the tissue retrieved from formalin was examined by electronmicroscopy in one case.

Results-The tumours were small and subcutaneous. Both showed features of benign schwannoma; one had a multinodular plexiform pattern. In addition, rosette-like structures consisting of collagenous cores surrounded by small round cells or slightly larger epithelioid cells were present. Tumour cells were positive for $\mathrm{S} 100$ protein, Leu 7, and in one case GFAP, but were negative for neurofilament protein, synaptophysin, and MIC2. Type IV collagen surrounded individual cells. Electronmicroscopy in case 2 confirmed schwannian features (lamina, processes) and failed to show features of neuroblastoma (neuroendocrine granules).
\end{abstract}

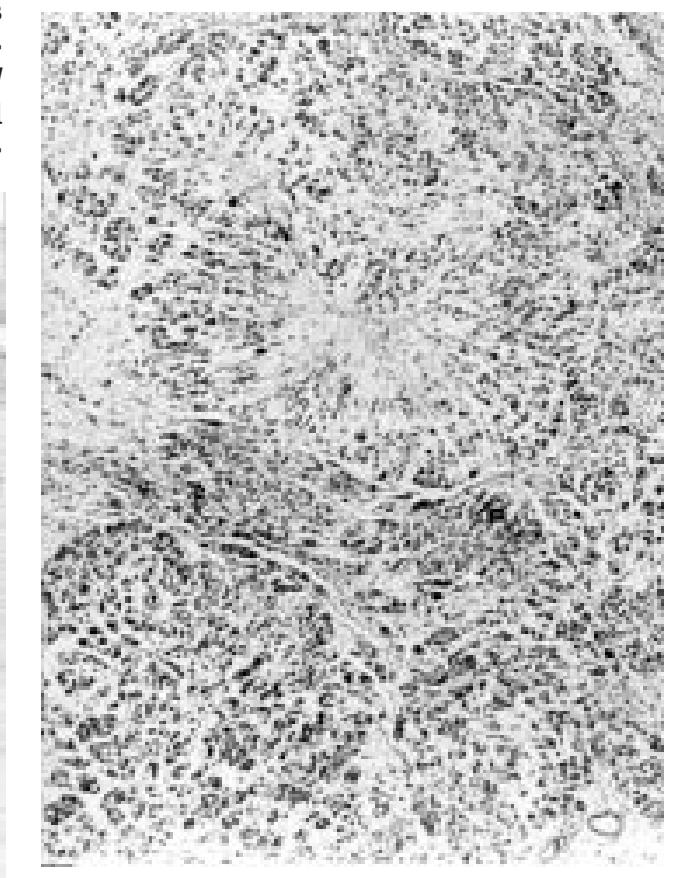

Figure 2 Lobulated tumour with rosette-like structures (case 2). Haematoxylin and eosin.

gists to avoid erroneous diagnoses and inappropriate treatment.

(f Clin Pathol 1998;51:842-845)

Keywords: schwannoma; neuroblastoma-like tumour; rosette formation

Schwannoma (neurilemmoma) is the most frequently encountered benign nerve sheath tumour and can present a wide spectrum of histological patterns, ranging from the typical schwannoma with bland spindle cells forming dense Antoni A and looser Antoni B areas to rarer forms like "ancient" schwannoma, plexiform schwannoma, schwannoma with glandular and pseudoglandular elements, epithelioid schwannoma, and schwannoma with neuroblastoma-like areas. ${ }^{1-7}$ To the best of our knowledge, only four cases of benign schwannoma with neuroblastoma-like areas have so far been reported. Epithelioid cells and neuroblastoma/peripheral primitive neuroectodermal tumour (PNET)-like areas may also be present in malignant peripheral nerve sheath tumours. ${ }^{18-11}$ Therefore, any epithelioid or neuroblastoma-like features in a neural lesion may suggest a more sinister pathology, and further careful evaluation of such cases becomes imperative. We describe two cases of benign 


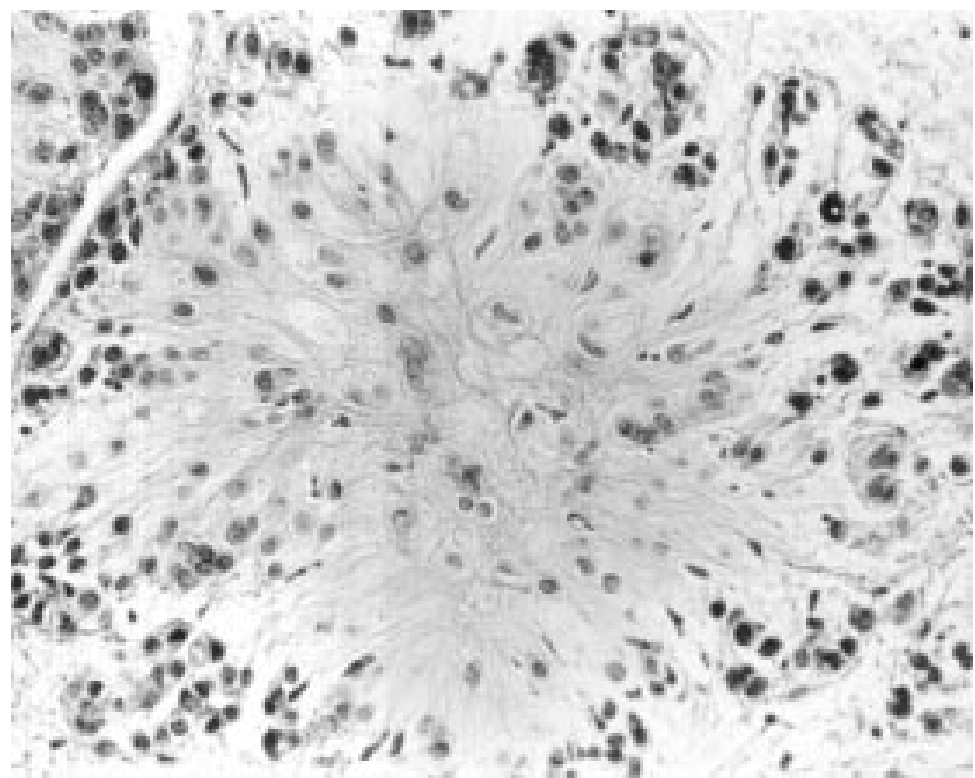

Figure 3 Fibrillar collagenous centre of a rosette-like structure (case 2). Haematoxylin and eosin.

schwannoma, both showing rosette-like structures mimicking neuroblastoma/PNET, one with focal epithelioid features in a plexiform schwannoma, the other showing extensive epithelioid change.

\section{Case histories}

CASE 1

A 53 year old woman presented with a painless soft/cystic lump in the left vulva. Three years previously she had had a total hysterectomy and bilateral salpingo-oophorectomy and omentectomy with appendicectomy for a Figo stage III adenocarcinoma involving both ovaries. In addition to well differentiated endometrioid adenocarcinoma of the ovaries, she also had an endometrioid type of endometrial adenocarcinoma and a carcinoid tumour of the appendix. The patient did not receive any

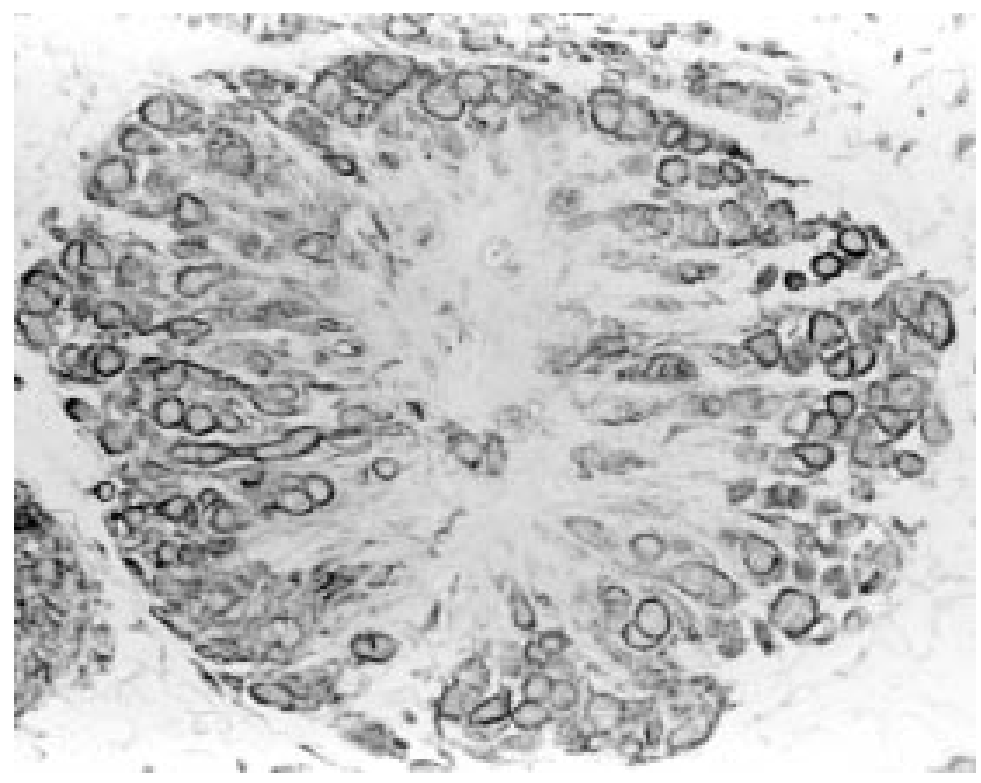

Figure 4 Immunohistochemical staining for type IV collagen around individual tumour cells. Streptavidin peroxidase method (case 2). radiotherapy and there was no evidence of neurofibromatosis. A $2.0 \mathrm{~cm}$ diameter vulval subcutaneous lesion was removed and subjected to histology to exclude a metastatic carcinoma. There was no evidence of recurrence or metastasis 18 months after excision.

\section{CASE 2}

A 35 year old woman presented with a painless lump near her left knee. There was no other significant history. A pale, well circumscribed subcutaneous nodule $(1.8 \mathrm{~cm}$ in diameter) was removed. The patient did not have any sign of neurofibromatosis. There was no evidence of recurrence or metastasis 24 months after excision.

\section{Methods}

LIGHT MICROSCOPY

Paraffin sections from both specimens were stained with haematoxylin and eosin, toluidineblue, Masson's trichrome, and van Gieson stains. They were also immunostained with primary antibodies to the following antigens, using the streptavidin-biotin-peroxidase method: S-100 protein, Leu-7, synaptophysin, neurofilament protein, glial fibrillary acidic protein (GFAP), NKIC3, smooth muscle actin (SMA), desmin (D33), type IV collagen, and Ewing's sarcoma marker MIC2.

\section{ELECTRON MICROSCOPY}

Tissue was retrieved from histological formalin in case 2. The sample was osmicated, stained with aqueous uranyl acetate, dehydrated in ethanol and propylene oxide, and embedded in epoxy resin. Cellular and myxoid areas of the tumour were identified on toluidine-blue stained resin sections. Ultrathin sections were stained in aqueous uranyl acetate and Reynolds' lead citrate. In addition, a wax section containing rosette-like areas was processed using the pop-off technique. ${ }^{12}$

\section{Pathological findings}

LIGHT MICROSCOPY

Case 1

The dermis and subcutis showed a well circumscribed and multinodular encapsulated lesion (fig 1) composed of plump spindle cells, rounded hyperchromatic cells with little cytoplasm, and epithelioid cells with moderate amounts of eosinophilic cytoplasm. Characteristic Antoni A and Antoni B areas were present. The former showed a few prominent rosettelike structures with fibrillary centres surrounded by small round cells with hyperchromatic nuclei and scanty cytoplasm. No mitoses were seen and there was no necrosis. There was no evidence of metastatic adenocarcinoma or carcinoid tumour.

\section{Case 2}

The biopsy comprised a well circumscribed and partly encapsulated lobulated tumour composed of small nests and groups of small round cells with scanty cytoplasm and epithelioid cells showing predominantly uniform 

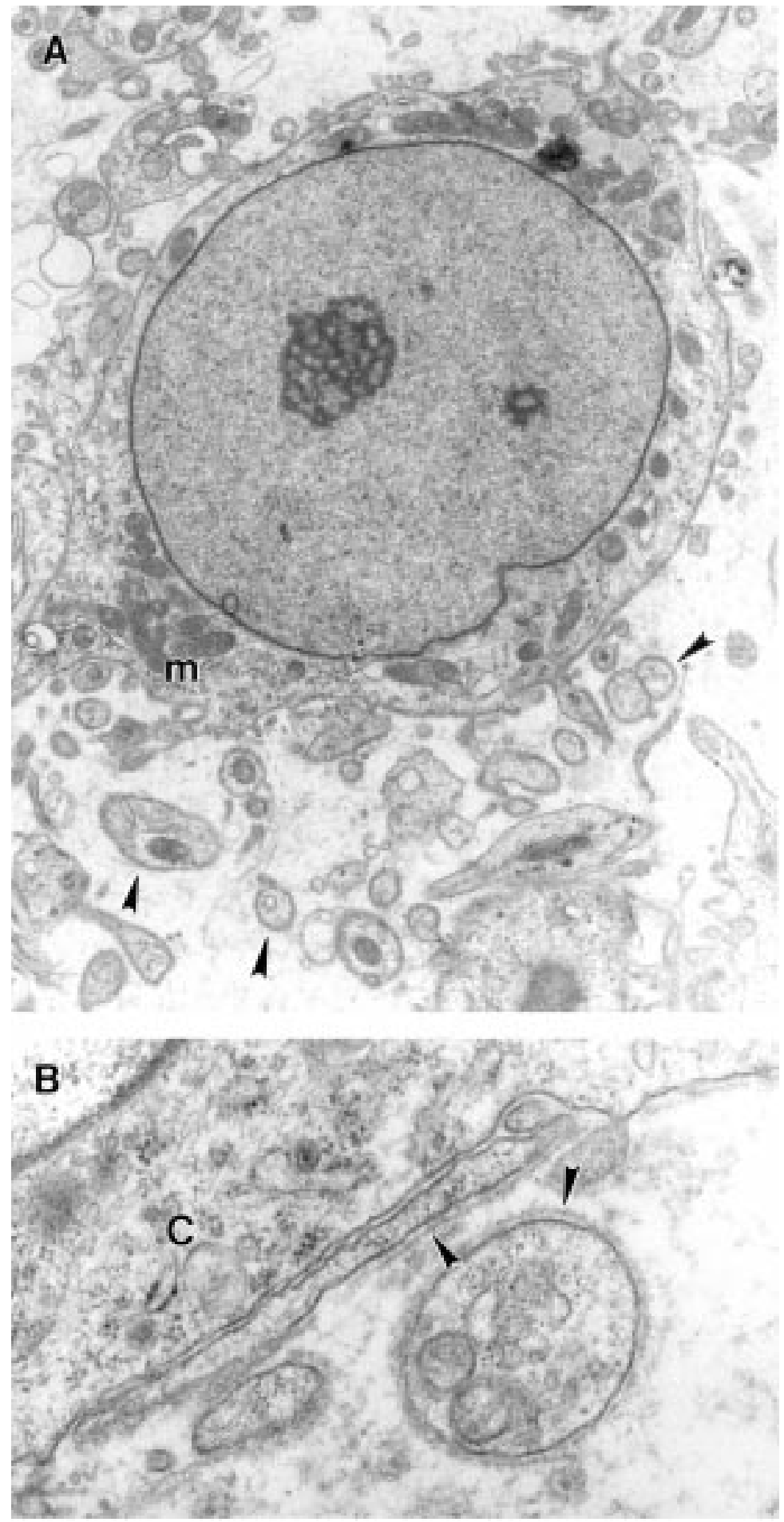

Figure 5 (A) Round tumour cell with cytoplasm containing fair numbers of mitochondria (m). Note processes (arrowheads). Magnification $\times 7800$ (case 2). (B) Detail of slender and rounded processes coated with lamina (arrowheads) close to a tumour cell (C). Magnification $\times 38900$ (case 2).

nuclei and eosinophilic or clear cytoplasm. Mild nuclear pleomorphism was noted and focally the nuclei showed prominent nucleoli. An occasional mitotic figure was present and no necrosis was seen. There were several prominent rosette-like structures with cells arranged around fibrillar collagenous centres (figs 2 and 3). Many hyalinised blood vessels were present and the stroma was markedly myxoid in places.
HISTOCHEMICAL FEATURES

Masson's trichrome and van Gieson stains showed presence of collagen in the centres of rosette-like areas in both cases but the positivity was weak in case 1 . Strong collagen staining was seen around the tumour cells in both cases.

\section{Immunohistochemistry}

There was strong S100 protein positivity and weak and focal Leu 7 positivity in the tumour cells in both cases. Type IV collagen was strongly positive around the tumour cells in both cases (fig 4) and in the centres of rosettelike areas in case 2 . The centres in case 1 were weakly positive. The tumour cells were strongly positive for GFAP in case 1 and negative in case 2 . All other immunostains were negative.

\section{Electronmicroscopy}

The tumour cells had euchromatic nuclei and well developed, often central, nucleoli. Cytoplasm was moderate in quantity and contained large numbers of mitochondria, sometimes in fields excluding other organelles (fig 5A). Also present were a few primary and secondary lysosomes, some lipid droplets, endocytosed collagen, and a fairly well developed Golgi apparatus. In the myxoid areas, mitochondria were fewer and there was more rough endoplasmic reticulum, suggesting matrix synthesis. A few dispersed intermediate filaments, presumably vimentin, were present between organelles. The cell surface had a well defined single lamina. Rounded or finger-like processes projecting from the cell surface were numerous, and were also covered with a lamina (fig 5B). In the formalin fixed specimen embedded for microscopy, the rosette-like areas were not encountered. The tissue examined from the pop-off technique was non-contributory through poor preservation.

\section{Discussion}

Goldblum et al first drew attention to a variant of schwannoma histologically mimicking a neuroblastoma or ependymoma by virtue of the presence of small round hyperchromatic Schwann cells with a high nucleocytoplasmic ratio, forming rosette-like structures around collagenous cores or blood vessels. ${ }^{6}$ They described three cases, two resembling neuroblastoma with Homer-Wright rosette-like structures and a third case showing perivascular ependymomatous rosettes. In all three, a diagnosis of benign schwannoma was confirmed on morphological, immunohistochemical, and ultrastructural grounds. In addition, Fisher et al described a case of epithelioid schwannoma containing neuroblastoma-like areas. $^{\text {? }}$

Malignant transformation of benign schwannoma, although extremely rare, has now been described in several cases. These usually take the form of malignant peripheral nerve sheath tumours (MPNST) of either conventional or epithelioid type, but occasional cases of neuroblastic malignancy have also been reported. ${ }^{8-10}$ Meis et al have also described similar epithelioid and neuroepithelial-like cells in malignant schwannomas (MPNST) in 
children. ${ }^{11}$ Therefore the presence of rosettelike areas, formed by epithelioid and/or round hyperchromatic cells, may result in diagnostic difficulties.

Our case 2 was very similar to the case described by Fisher et $a l,{ }^{7}$ but a case of plexiform schwannoma showing a combination of neuroblastoma-like areas and focal epithelioid change (case 1) has not been described before. Histologically, the features which favoured a benign diagnosis in our cases were encapsulation, lack of significant mitotic activity, and absence of necrosis. Although the rosettes in these cases resembled HomerWright rosettes, they were much larger than those seen in a neuroblastoma. Also, their centres appeared to be composed of collagen rather than neurites, as demonstrated by histochemical stains and immunohistochemistry. Although the lesional cells were small and round in places in case 2 and were reminiscent of neuroblastoma cells, they were positive for S100 protein and negative for neuronal markers synaptophysin and neurofilament protein. Moreover, individual cells were surrounded by type IV collagen - a feature characteristically seen in schwannian but not in neuronal tumours. ${ }^{13}$ MIC 2 negativity confirmed an absence of true PNET type differentiation. In case 2, electronmicroscopy further confirmed the tumour cells to be schwannian by showing prominent lamina and cell processes. A lack of neuroendocrine granules favoured a diagnosis of Schwann cell tumour over neuroblastoma.

With regard to the GFAP staining, while this is known to be a consistent marker of ependymomas, it has also been found in $33 \%$ of benign schwannomas in one series. ${ }^{14}$ On its own, therefore, GFAP should not be regarded as indicating ependymomatous differentiation, especially in the present context in which other features favoured schwannian differentiation.

In summary, these rather unusual tumours showed some features on routine histology suggestive of malignancy and therefore requiring further assessment. Ancillary techniques confirmed schwannian differentiation and excluded neuroblastoma.

1 Enzinger FM, Weiss SW. Benign tumors of peripheral nerves. In: Soft tissue tumors, 3rd ed. St Louis: CV Mosby, 1995:821-88.

2 Fletcher CDM, Davies SE. Benign plexiform (multinodular) schwannoma: a rare tumour unassociated with neurofilar) schwannoma: a rare tumour unassociate

3 Brooks JJ, Draffen RM. Benign glandular schwannoma. Brooks JJ, Draffen RM. Benign gland
Arch Pathol Lab Med 1992;116:192-5.

4 Ferry JA, Dickersin GR. Pseudoglandular schwannoma. Am f Clin Pathol 1988;89:546-52.

5 Chan JKC, Fok KO. Pseudoglandular schwannoma. Histopathology 1996;29:481-3.

6 Goldblum JR, Beals TF, Weiss SW. Neuroblastoma-like neurilemmoma. Am f Surg Pathol 1994;18:266-73.

7 Fisher C, Chappel ME, Weiss SW. Neuroblastoma-like epithelioid schwannoma. Histopathology 1995;26:193-4.

8 Carstens PHB, Schrodt G. Malignant transformation of a benign encapsulated neurilemmoma. Am $\mathcal{F}$ Clin Pathol 1969;51:144-9.

9 Yousem SA, Colby TV, Urich H. Malignant epithelioid schwannoma arising in a benign schwannoma. Cancer 1985;55:2799-803.

10 Rasbridge SA, Browse NL, Tighe JR, et al. Malignant nerve sheath tumour arising in a benign ancient schwannoma. Histopathology 1989;14:525.

11 Meis JM, Enzinger FM, Martz KL, et al. Malignant periphral nerve sheath tumours (malignant schwannomas) in children. Am F Surg Pathol 1992;16:694-707.

12 Bretscheider A, Burns W, Morrison A. "Pop-off” technique. The ultra-structure of paraffin-embedded sections. $A m \mathcal{F}$ Clin Pathol 1981;76:450-3.

13 Leong A S, Vinyurat S, Suthipinta Wong C, et al. Patterns of basal lamina immunostaining in soft tissue and bony tumours. Appl Immunohistochem 1997;5:1-7.

14 Grey MH, Rosenberg AE, Dickersin GR, et al. Hum Pathol 1989;20:1089-96. 